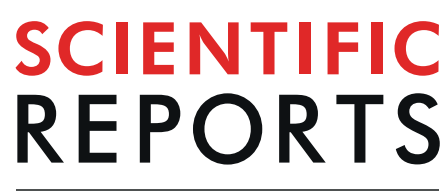

natureresearch

\title{
OPEN The FTO gene polymorphism rs9939609 is associated with obesity and disability in multiple sclerosis patients
}

\begin{abstract}
Ahmad Al-Serri ${ }^{1 *}$, Raed Alroughani ${ }^{2}$ \& Rabeah A. Al-Temaimi ${ }^{1}$
Obesity is a well-known risk factor for multiple diseases including multiple sclerosis (MS). Polymorphisms in the fat-mass obesity (FTO) gene have been consistently found to be associated with obesity, and recently found to increase the risk of developing MS. We therefore assessed the common FTO gene polymorphism (rs9939609) in relation to obesity, risk of developing MS and its disability in a cohort of MS patients. A cohort of 200 MS patients ( 135 females and 65 males) were genotyped for the FTO rs9939609 polymorphism. Using both logistic and linear regression we assessed the relationship between the variant and the selected phenotypes under both an additive and recessive genetic models. The A-allele was found to be associated with being overweight/obese in MS patients (OR $=2.48$ ( $95 \%$ $\mathrm{Cl}$ 1.17-5.29); $\mathrm{p}=0.01)$. In addition, The A-allele was also found to be associated with increased MS disability $(\beta=0.48(95 \% \mathrm{Cl} 0.03-0.92) ; p=0.03)$. However, no association was found with risk of developing MS ( $p>0.05)$. Moreover, our association with obesity is consistent with previous reports, whereas the association with disability is novel and warrants further investigation on the role of FTO in disease progression.
\end{abstract}

Multiple sclerosis (MS) is a chronic demyelinating autoimmune disorder of the central nervous system with over 2.2 million people affected worldwide ${ }^{1}$. The average MS age of onset is between 20-40 years of age, however MS has been reported to occur in younger and older ages as well ${ }^{2,3}$. There is no specific etiology for the development of MS, however both genetic and environmental factors have been found to contribute to its development ${ }^{4,5}$. Obesity is an environmental risk factor for multiple diseases including $\mathrm{MS}^{6-8}$. These studies have been conducted on both children and adults and have shown a 2-fold increase in the development of MS in obese (Body mass index $(B M I)>30 \mathrm{~kg} / \mathrm{m}^{2}$ ) subjects compared to subjects with BMI between $18.5-21 \mathrm{~kg} / \mathrm{m}^{2}$. In addition, obesity has been shown to associate with worsening MS disability, and a faster conversion of relapsing-remitting MS (RRMS) to secondary progressive MS (SPMS) ${ }^{10-12}$. With studies implicating the low-grade neuroinflammation associated with obesity to contribute to MS disability progression ${ }^{13,14}$. MS disability monitoring measures the change in disability over time in MS patients that is an important guide for clinicians to stratify patients progress, make disease management decisions and early therapeutic interventions. The expanded disability status scale (EDSS) has been used extensively since its development to guide in diagnosing MS disease clinical course and designing and monitoring treatment regimens to slow down MS disease progression ${ }^{15}$.

The prevalence of obesity has tripled in the past 30 years and it is estimated to be $50 \%$ in the Kuwaiti popula$\operatorname{tion}^{16}$. Both genetic and environmental factors contribute to the development of obesity ${ }^{17}$. In 2007 a genome-wide association study (GWAS) found a single nucleotide polymorphism (SNP) in the fat mass obesity (FTO) gene to be associated with increased BMI in both children and adults ${ }^{18,19}$. The variant (rs9939609) today has been consistently associated with obesity in multiple populations making FTO the most commonly studied obesity gene $^{18,20}$. The FTO is an alpha-ketoglutarate dependent dioxygenase that is highly expressed in the nucleus. While the exact function of this protein is unknown it is reported to be an RNA demethylase that mediates demethylation of different RNA species including mRNAs, tRNAs and snRNAs ${ }^{21,22}$. However, the most studied role of FTO is as a regulator of fat mass, adipogenesis and energy homeostasis ${ }^{23}$. FTO is believed to be involved in multiple

${ }^{1}$ Human Genetics Unit, Department of Pathology, Faculty of Medicine, Kuwait University, Kuwait, PO Box 24923, Safat, 13110, Kuwait. 'Division of Neurology, Department of Medicine, Amiri Hospital, Kuwait City, Kuwait. *email: Ahmad.Alserri@hsc.edu.kw 


\begin{tabular}{|c|c|c|c|}
\hline Criteria & $\begin{array}{l}\text { MS cohort } \\
(\mathrm{n}=200)\end{array}$ & $\begin{array}{l}\text { Controls } \\
(n=206)\end{array}$ & p-value \\
\hline \multicolumn{4}{|l|}{$\operatorname{Sex}(\%)$} \\
\hline Female & $135(67.5)$ & $125(60.5)$ & 0.152 \\
\hline Male & $65(32.5)$ & $81(39.5)$ & \\
\hline $\begin{array}{l}\text { Age in years (Average } \\
\left.\left[ \pm \mathrm{SD}^{\mathrm{a}}\right]\right)\end{array}$ & $32.32 \pm 9.35$ & $52.39 \pm 8.28$ & $<0.0001$ \\
\hline $\mathrm{BMI}^{\mathrm{b}}$ (Average $\left.[ \pm \mathrm{SD}]\right)$ & $27.32 \pm 6.29$ & $31.52 \pm 7.14$ & $<0.0001$ \\
\hline \multicolumn{4}{|l|}{ BMI categories (\%) } \\
\hline Underweight & $9(4.5)$ & $0(0)$ & $<0.0001$ \\
\hline Normal weight & $73(36.5)$ & $24(11.5)$ & \\
\hline Overweight/Obese & $118(59)$ & $182(89.5)$ & \\
\hline MS type (\%) & & NA & \\
\hline Benign MS & $9(4.5)$ & - & \\
\hline $\mathrm{RRMS}^{\mathrm{c}}$ & $171(85.5)$ & - & \\
\hline SPMS $^{\mathrm{d}}$ & $17(8.5)$ & - & \\
\hline PPMS $^{\mathrm{e}}$ & $3(1.5)$ & - & \\
\hline EDSS $^{f}($ Average $[ \pm S D])$ & $2.377 \pm 1.75$ & NA & \\
\hline $\begin{array}{l}\text { Disease duration in } \\
\text { years (Average }[ \pm \mathrm{SD}] \text { ) }\end{array}$ & $5.69 \pm 5.22$ & NA & \\
\hline
\end{tabular}

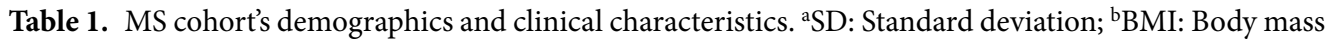

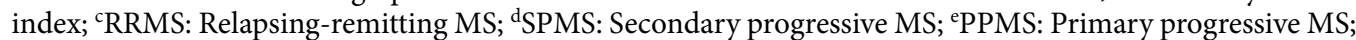
fEDSS: Expanded disability status scale; NA: Not applicable.

\begin{tabular}{|c|c|c|c|c|c|}
\hline SNP & Model & Controls & MS & $\mathrm{OR}^{\mathrm{a}}\left(95 \% \mathrm{CI}^{\mathrm{b}}\right)$ & p-value \\
\hline \multirow{7}{*}{ FTO rs9939609 } & \multicolumn{5}{|l|}{ Additive } \\
\hline & TT & $62(30.1 \%)$ & $59(29.5 \%)$ & & \\
\hline & TA & $97(47.1 \%)$ & $94(47 \%)$ & $1.25(0.8-1.94)$ & 0.33 \\
\hline & AA & $47(22.8 \%)$ & $47(23.5 \%)$ & & \\
\hline & \multicolumn{5}{|c|}{ Recessive } \\
\hline & TT/TA & $159(77.2 \%)$ & $153(76.5 \%)$ & & \\
\hline & AA & $47(22.8 \%)$ & $47(23.5 \%)$ & $\begin{array}{l}1.57 \\
(0.74-3.32)\end{array}$ & 0.23 \\
\hline
\end{tabular}

Table 2. Assessing the risk of developing MS by comparing the FTO polymorphism between healthy controls and MS patients under both an additive and a recessive genetic model. ${ }^{\mathrm{a}} \mathrm{OR}$ : Odds ratio; ${ }^{\mathrm{b}} \mathrm{CI}$ : Confidence interval.

pathways including; DNA/RNA repair ${ }^{24}$, adipogenesis ${ }^{25}$, thermogenesis ${ }^{26}$, and neurogenesis ${ }^{27}$. FTO genetic variants and altered expression have been reported to associate with several obesity-related chronic diseases such as type-2-diabetes ${ }^{28}$ and cancer ${ }^{29}$. In addition, FTO genetic variants have been found to affect disease outcome and risk in obese patients with other diseases such as psoriasis ${ }^{30}$, polycystic ovaries syndrome ${ }^{31}$, and cardiovascular diseases $^{32}$. Interestingly, only two studies to date have investigated FTO variants in MS patients ${ }^{9,33}$.

Based on the association of obesity with MS risk and the established association of FTO rs9939609 with obesity, investigating obesity genes in relation to MS should be conducted to understand the mechanism underlying the link between the two diseases. Therefore, in this study we were interested in assessing the relationship between the common FTO gene polymorphism (rs9939609) with obesity, MS risk and disability in a cohort of MS patients.

\section{Results}

Assessing rs9939609 in relation to MS risk and BMI status in MS patients. The MS population sample in this study included 135 females and 65 males $(n=200)$, whereas healthy controls included 125 females and 91 males $(\mathrm{n}=206)$. The demographics and clinical characteristics are shown in Table 1.

FTO rs9939609 genotype distribution in the MS cohort did not differ from the sampled healthy Kuwaiti population distributions after adjusting for both age and gender under both an additive and recessive genetic model ( $\mathrm{p}=0.33,0.23$ respectively) (Table 2 ). In addition, healthy controls and MS cohort rs9939609 genotype distribution were in Hardy-Weinberg Equilibrium ( $\mathrm{p}=0.44$ and 0.42 , respectively).

We assessed the relationship between the common FTO gene polymorphism and obesity status in MS patients after adjusting for both age and gender (Table 3 ). In a dose-dependent trend the A-allele was found to be associated with overweight/obesity $(\mathrm{p}=0.04)$. Moreover, using a recessive genetic model a significant difference $(\mathrm{p}=0.01)$ was observed in the distribution of the AA genotype in the overweight/obese group compared to the BMI normal group, $29.9 \%$ compared to $14.5 \%$ respectively (Table 3 ). 


\begin{tabular}{|c|c|c|c|c|c|}
\hline SNP & Model & Normal weight & Overweight/obese & $\mathrm{OR}^{\mathrm{a}}\left(95 \% \mathrm{CI}^{\mathrm{b}}\right)$ & p-value \\
\hline \multirow{7}{*}{ FTO rs9939609 } & \multicolumn{5}{|l|}{ Additive } \\
\hline & TT & $28(33.7 \%)$ & $31(26.5 \%)$ & & \\
\hline & $\mathrm{TA}$ & $43(51.8 \%)$ & $51(43.6 \%)$ & $1.51(1-2.28)$ & 0.04 \\
\hline & $\mathrm{AA}$ & $12(14.5 \%)$ & 35 (29.9\%) & & \\
\hline & \multicolumn{5}{|c|}{ Recessive } \\
\hline & TT/TA & $71(85.5 \%)$ & $82(70.1 \%)$ & & \\
\hline & AA & $12(14.5 \%)$ & 35 (29.9\%) & $2.48(1.17-5.29)$ & 0.01 \\
\hline
\end{tabular}

Table 3. Relationship between the FTO polymorphism and obesity status in MS patients using both an additive and a recessive genetic model. ${ }^{\mathrm{a} O R}$ : Odds ratio; ${ }^{\mathrm{b}} \mathrm{CI}$ : Confidence interval.

\begin{tabular}{|l|l|l|l|l|}
\hline SNP & Model & EDSS $^{\mathbf{a}}$ & $\boldsymbol{\beta}^{\mathbf{b}}\left(\mathbf{9 5} \% \mathbf{C I}^{\mathbf{c}}\right)$ & p-value \\
\hline \multirow{5}{*}{ FTO rs9939609 } & Additive & \multicolumn{3}{|l|}{} \\
\cline { 2 - 5 } & TT & $2.09 \pm 0.21$ & & \\
\cline { 2 - 5 } & TA & $2.18 \pm 0.16$ & $0.26(0.009-0.52)$ & 0.04 \\
\cline { 2 - 5 } & AA & $2.72 \pm 0.31$ & & \\
\cline { 2 - 5 } & Recessive & \\
\cline { 2 - 5 } & TT/TA & $2.15 \pm 0.12$ & & \\
\cline { 2 - 5 } & AA & $2.72 \pm 0.31$ & $0.48(0.03-0.92)$ & 0.03 \\
\hline
\end{tabular}

Table 4. Relationship between the FTO polymorphism and EDSS using both additive and recessive genetic

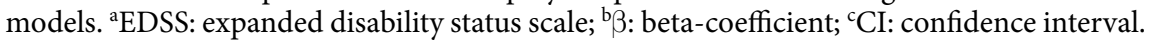

Assessing the relationship between rs9939609 and EDSS. The relationship between the common FTO gene polymorphism and EDSS showed a significant dose-dependent association $(\mathrm{p}=0.04)$ when adjusted for gender and disease duration (Table 4). A recessive genetic model showed carriers of the AA genotype had 0.67 higher EDSS compared to those with both TT and TA genotypes combined $(\mathrm{p}=0.03)$ (Table 4$)$. The association remained significant after adjusting for gender, disease duration, age and BMI categories ( $p=0.037$ for the additive model and $\mathrm{p}=0.025$ for the recessive model).

\section{Discussion}

In this study we report an association between FTO rs9939609 gene polymorphism and risk of obesity in a cohort of MS patients. In addition, we found the FTO risk allele to associate with increase in disability of MS patients. Although we did not find an association between the FTO variant and risk of developing MS when compared to controls, our finding is consistent with the previous study showing no association between FTO rs9939609 in MS patients ${ }^{33}$. The study by Davis et al., reported the risk allele of rs9939609 not to be associated with MS risk however is associated with increased homocysteine levels in MS patients compared to controls ${ }^{33}$. However, a recent study by Gianfrancesco et al., found a direct effect between another FTO variant (rs1558902) and risk of developing MS in Hispanics ${ }^{9}$. Therefore, our inconsistency and lack of association between the FTO rs9939609 and MS risk can be due to the differences in the variant selected and the population studied. Being overweight and obese is endemic to the Kuwaiti population, specifically in Kuwaiti nationals; which potentiates a significant health risk and predisposition to obesity-related diseases in the population ${ }^{34}$. Several studies have repeatedly shown that obesity is a risk factor for early-onset $\mathrm{MS}^{35-37}$. While the underlying mechanism by which obesity contributes to MS etiology remains unclear several hypotheses have been postulated including; the effect of persistent low-grade neuroinflammation ${ }^{38}$, the role of adipose tissue adipokines in promoting inflammation ${ }^{39}$, and obesity-related central nervous system cardiovascular alterations ${ }^{40}$. The role of obesity in MS expands beyond risk as it has been shown to worsen MS disease disability and progression as well ${ }^{10,13}$.

FTO rs9939609 has consistently been shown to associate with higher BMI across different populations including the Kuwaiti population ${ }^{18,20}$. The effects of rs 9939609 has been rigorously investigated since its discovery for its functional impact in relation to obesity. Reported evidence points to a role in food craving behavior since FTO is highly expressed in the hypothalamus that controls the regulation of food intake ${ }^{41,42}$. Another evidence suggests rs9939609 has direct influence on muscle mass and oxidative potential as it was found associated with decreased slow-twitch muscle fibers in lean sport athletes and lower muscle lean mass in non-resistance trained athletes ${ }^{43,44}$. In addition, it has been shown that MS affects skeletal muscles resulting in fewer and smaller muscle fibers and lower lean muscle mass ${ }^{44}$. Collectively, rs 9939609 functional roles may explain the association of the A allele with MS disability. It is plausible that MS carriers of the A allele have less cognitive restraint towards food intake and physical activity commitment. Lower cognitive restraint may impact an already poor lean muscle mass in these patients reducing their inclination to perform physical exercise. This possibility is amplified by the psychological impact of their MS diagnosis which may ultimately result in increases in body fat and less physical activity that has been shown to directly impact disability in MS patients ${ }^{45,46}$. Moreover, the average EDSS score increase for all RRMS patients with EDSS scores between $0-6$ has been reported to be 0.168 per year suggesting that A allele 
carriers might not be recognized at risk of rapid disability development at the lower EDSS points (0-3) but are at a significant risk at higher ends of the scale $(>3.5)^{47}$. The clinical implication of A allele carriers having an 0.67 increase in EDSS after adjusting for disease duration should guide the clinical design of treatment regimens for this specific subset of MS patients to counter its influence early on MS progression as timing is of importance in MS disease clinical management ${ }^{48}$.

Interestingly, recent evidence of FTO's role in adult neurogenesis and the association of an FTO variant (rs1558902) with MS risk points to a possible direct neuronal function for FTO in MS relapse-repair and remyelination $^{9,27}$. Moreover, the study by Smemo et al., demonstrates a connection between FTO variants and the homeobox $I R X 3$ gene which is a transcription factor that is highly expressed in the brain ${ }^{49}$. The IRX3 gene is involved in body mass and composition, and is suggested to be regulated by FTO variants ${ }^{49}$.

Although clinical data was well defined and available for this MS cohort however the current study has its limitations. The modest sample size suggests the importance of replication studies on a larger and independent cohort before a putative conclusion on the role of FTO in MS progression can be drawn.

In conclusion, this is the first study as far as we are aware to report MS patients carrying FTO's risk allele are at a greater risk of MS disability progression and therefore should be encouraged to adapt to higher cognitive restrains towards food craving and higher commitment towards physical activity for better disease outcomes.

\section{Methods}

Patients sample selection and ethics. Two hundred Kuwaiti MS patients were recruited for this study at Dasman diabetes institute MS clinic. The study protocols were approved by Dasman diabetes institute ethical review committee and Health Sciences Center ethical committee, Kuwait, both of which adhere to the declaration of Helsinki Ethical Principles for Medical Research Involving Human Subjects. All study protocols and objectives were fully explained to all participants before securing informed written consent. MS patients' inclusion criteria were as follows; having a confirmed MS diagnosis according to the standardized modified McDonald's criteria ${ }^{50}$, a detailed clinical history (demographics, BMI measurement, age of MS onset, disease duration, expanded disability status scale (EDSS) score, and treatment history), being a Kuwaiti citizen, and an MS disease duration of $\geq 2$ years. All magnetic resonance imaging (MRI) and EDSS assessments were conducted by an experienced neurologist in accordance with the standard approved EDSS method ${ }^{51}$. A $4 \mathrm{~mL}$ blood sample was collected from each patient in an EDTA coated vacutainer. Collected blood samples were centrifuged at 2,500 $\times \mathrm{g}$ at room temperature for 10 minutes and buffy coat fractions were collected and stored at $-20^{\circ} \mathrm{C}$ until use.

FTO rs9939609 genotyping. Qiagen DNA mini kit (Qiagen, CA, USA) was used for buffy coat DNA extraction with minor modifications. In summary, 200 stored frozen buffy coat samples from MS patients were thawed in a water bath set at $37^{\circ} \mathrm{C}$, and $200 \mu \mathrm{l}$ aliquots were transferred to a sterile microcentrifuge tube. Samples were digested at $56^{\circ} \mathrm{C}$ with proteinase $\mathrm{K}$ and lysis buffer for 20 minutes. Absolute ethanol was added to stop sample digestion and precipitate proteins. Samples were eluted through a QIAamp mini spin filter columns to bind isolated DNA to the filter membranes. Columns were washed twice with wash buffers of differing stringency, and dried by spinning at $14,000 \times \mathrm{g}$ for one minute followed by air drying for 2 minutes. DNA was eluted with $150 \mu$ l of Tris-EDTA buffer. All centrifugations were performed at room temperature. DNA yield and quality were assessed using a NanoDrop spectrophotometer. FTO SNP rs9939609 was assessed using Taqman rs9939609 genotyping assay according to standard manufacturer protocol (Life Technologies, CA, USA). In brief, 50 ng of DNA was used in a total of $20 \mu \mathrm{l}$ reaction mixture inclusive of 1x TaqMan Universal PCR Master Mix and 1x probes/ primers mix. Reactions were loaded in a 96-well plate and a plate pre-PCR read was recorded in an ABI 7500 Fast Real-time PCR system (Life technologies, CA, USA). PCR was performed under the following conditions; a hold step at $95^{\circ} \mathrm{C}$ for 10 , and 45 cycles of denaturation at $92^{\circ} \mathrm{C}$ for 15 seconds followed by annealing/extension at $60^{\circ} \mathrm{C}$ for 1 minute. Post-PCR read was recorded for end-point fluorescence detection. Allelic discrimination analysis was performed and analyzed using ABI 7500 Fast Real-time PCR system SDS software (Life technologies, CA, USA).

Statistical analysis. FTO rs9939609 Kuwaiti population frequencies from a previous study were used to compare to MS cohort allelic and genotype distributions ${ }^{20}$. A subset of healthy Kuwaitis was selected from that study's population according to the following criteria; no history of any chronic disease (i.e. diabetes), being $\geq 40$ years old to exclude individuals that may have a future MS diagnosis, and a comparable sex ratio to our MS cohort. MS patients characteristics were expressed as mean \pm standard deviation (SD) and percentages where appropriate. Hardy-Weinberg equilibrium (HWE) was calculated. Association between the variant and MS risk, obesity status was assessed using binary logistic regression whereas linear regression was used to assess the association with EDSS expressed as odds ratio (OR) and Beta-coefficient $(\beta)$ respectively. Both an additive and recessive genetic models were used to assess the dose-dependent association and the impact of having two risk-alleles. All statistical analyses were performed using SPSS software (version 25; SPSS Inc, Chicago, IL, USA) and "SNPassoc" package from $\mathrm{R}$ where appropriate ${ }^{52}$. Significance was set at $\mathrm{p}<0.05$.

\section{Data availability}

The raw genotypic data is available for any meta-analysis studies upon request.

Received: 30 August 2019; Accepted: 2 December 2019;

Published online: 13 December 2019 


\section{References}

1. Collaborators, G. B. D. M. S. Global, regional, and national burden of multiple sclerosis 1990-2016: a systematic analysis for the Global Burden of Disease Study 2016. Lancet Neurol 18, 269-285, https://doi.org/10.1016/S1474-4422(18)30443-5 (2019).

2. Hansen, M. R. \& Okuda, D. T. Multiple Sclerosis in the Contemporary Age: Understanding the Millennial Patient with Multiple Sclerosis to Create Next-Generation Care. Neurol Clin 36, 219-230, https://doi.org/10.1016/j.ncl.2017.08.012 (2018).

3. Reinhardt, K., Weiss, S., Rosenbauer, J., Gartner, J. \& von Kries, R. Multiple sclerosis in children and adolescents: incidence and clinical picture - new insights from the nationwide German surveillance (2009-2011). Eur J Neurol 21, 654-659, https://doi. org/10.1111/ene.12371 (2014).

4. Axisa, P. P. \& Hafler, D. A. Multiple sclerosis: genetics, biomarkers, treatments. Curr Opin Neurol 29, 345-353, https://doi. org/10.1097/WCO.0000000000000319 (2016).

5. Jorg, S. et al. Environmental factors in autoimmune diseases and their role in multiple sclerosis. Cell Mol Life Sci 73, 4611-4622, https://doi.org/10.1007/s00018-016-2311-1 (2016).

6. Munger, K. L., Chitnis, T. \& Ascherio, A. Body size and risk of MS in two cohorts of US women. Neurology 73, 1543-1550, https:// doi.org/10.1212/WNL.0b013e3181c0d6e0 (2009).

7. Hedstrom, A. K., Olsson, T. \& Alfredsson, L. High body mass index before age 20 is associated with increased risk for multiple sclerosis in both men and women. Mult Scler 18, 1334-1336, https://doi.org/10.1177/1352458512436596 (2012).

8. Langer-Gould, A., Brara, S. M., Beaber, B. E. \& Koebnick, C. Childhood obesity and risk of pediatric multiple sclerosis and clinically isolated syndrome. Neurology 80, 548-552, https://doi.org/10.1212/WNL.0b013e31828154f3 (2013).

9. Gianfrancesco, M. A. et al. Causal Effect of Genetic Variants Associated With Body Mass Index on Multiple Sclerosis Susceptibility. Am J Epidemiol 185, 162-171, https://doi.org/10.1093/aje/kww120 (2017).

10. Pilutti, L. A. \& Motl, R. W. Body composition and disability in people with multiple sclerosis: A dual-energy x-ray absorptiometry study. Mult Scler Relat Disord 29, 41-47, https://doi.org/10.1016/j.msard.2019.01.009 (2019).

11. Fitzgerald, K. C. et al. Measures of general and abdominal obesity and disability severity in a large population of people with multiple sclerosis. Mult Scler, 1352458519845836, https://doi.org/10.1177/1352458519845836 (2019).

12. Manouchehrinia, A. et al. Association of Pre-Disease Body Mass Index With Multiple Sclerosis Prognosis. Frontiers in neurology 9 , 232, https://doi.org/10.3389/fneur.2018.00232 (2018).

13. Stampanoni Bassi, M. et al. Obesity worsens central inflammation and disability in multiple sclerosis. Mult Scler, 1352458519853473, https://doi.org/10.1177/1352458519853473 (2019).

14. Castro, K. et al. Body Mass Index in Multiple Sclerosis modulates ceramide-induced DNA methylation and disease course. EBioMedicine 43, 392-410, https://doi.org/10.1016/j.ebiom.2019.03.087 (2019).

15. Cinar, B. P. \& Yorgun, Y. G. What We Learned from The History of Multiple Sclerosis Measurement: Expanded Disability Status Scale. Noro psikiyatri arsivi 55, S69-S75, https://doi.org/10.29399/npa.23343 (2018).

16. Ng, M. et al. Global, regional, and national prevalence of overweight and obesity in children and adults during 1980-2013: a systematic analysis for the Global Burden of Disease Study 2013. Lancet 384, 766-781, https://doi.org/10.1016/S01406736(14)60460-8 (2014).

17. Heianza, Y. \& Qi, L. Impact of Genes and Environment on Obesity and Cardiovascular Disease. Endocrinology 160, 81-100, https:// doi.org/10.1210/en.2018-00591 (2019).

18. Frayling, T. M. et al. A common variant in the FTO gene is associated with body mass index and predisposes to childhood and adult obesity. Science 316, 889-894, https://doi.org/10.1126/science.1141634 (2007).

19. Scuteri, A. et al. Genome-wide association scan shows genetic variants in the FTO gene are associated with obesity-related traits. PLoS Genet 3, e115, https://doi.org/10.1371/journal.pgen.0030115 (2007).

20. Al-Serri, A. et al. Association of FTO rs9939609 with Obesity in the Kuwaiti Population: A Public Health Concern? Med Princ Pract 27, 145-151, https://doi.org/10.1159/000486767 (2018).

21. Bartosovic, M. et al. N6-methyladenosine demethylase FTO targets pre-mRNAs and regulates alternative splicing and 3'-end processing. Nucleic Acids Res 45, 11356-11370, https://doi.org/10.1093/nar/gkx778 (2017).

22. Mauer, J. et al. FTO controls reversible m(6)Am RNA methylation during snRNA biogenesis. Nat Chem Biol 15, 340-347, https:// doi.org/10.1038/s41589-019-0231-8 (2019).

23. Mizuno, T. M. Fat Mass and Obesity Associated (FTO) Gene and Hepatic Glucose and Lipid Metabolism. Nutrients 10, https://doi. org/10.3390/nu10111600 (2018).

24. Fedeles, B. I., Singh, V., Delaney, J. C., Li, D. \& Essigmann, J. M. The AlkB Family of Fe(II)/alpha-Ketoglutarate-dependent Dioxygenases: Repairing Nucleic Acid Alkylation Damage and Beyond. J Biol Chem 290, 20734-20742, https://doi.org/10.1074/jbc. R115.656462 (2015).

25. Ronkainen, J. et al. Fat mass- and obesity-associated gene Fto affects the dietary response in mouse white adipose tissue. Sci Rep $\mathbf{5}$, 9233, https://doi.org/10.1038/srep09233 (2015).

26. Claussnitzer, M. et al. FTO Obesity Variant Circuitry and Adipocyte Browning in Humans. N Engl J Med 373, 895-907, https://doi. org/10.1056/NEJMoa1502214 (2015).

27. Li, L. et al. Fat mass and obesity-associated (FTO) protein regulates adult neurogenesis. Hum Mol Genet 26, 2398-2411, https://doi. org/10.1093/hmg/ddx128 (2017).

28. Wang, Q. et al. Relationship between fat mass and obesity-associated gene expression and type 2 diabetes mellitus severity. Exp Ther Med 15, 2917-2921, https://doi.org/10.3892/etm.2018.5752 (2018).

29. Huang, X., Zhao, J., Yang, M., Li, M. \& Zheng, J. Association between FTO gene polymorphism (rs9939609 T/A) and cancer risk: a meta-analysis. Eur J Cancer Care (Engl) 26, https://doi.org/10.1111/ecc.12464 (2017).

30. Tupikowska-Marzec, M. et al. The Influence of FTO Polymorphism rs 9939609 on Obesity, Some Clinical Features, and Disturbance of Carbohydrate Metabolism in Patients with Psoriasis. Biomed Res Int 2019, 7304345, https://doi.org/10.1155/2019/7304345 (2019).

31. Liu, A. L. et al. Association between fat mass and obesity associated (FTO) gene rs9939609 A/T polymorphism and polycystic ovary syndrome: a systematic review and meta-analysis. BMC Med Genet 18, 89, https://doi.org/10.1186/s12881-017-0452-1 (2017).

32. Liu, C., Mou, S. \& Pan, C. The FTO gene rs 9939609 polymorphism predicts risk of cardiovascular disease: a systematic review and meta-analysis. PLoS One 8, e71901, https://doi.org/10.1371/journal.pone.0071901 (2013).

33. Davis, W. et al. The fat mass and obesity-associated FTO rs 9939609 polymorphism is associated with elevated homocysteine levels in patients with multiple sclerosis screened for vascular risk factors. Metab Brain Dis 29, 409-419, https://doi.org/10.1007/s11011014-9486-7 (2014).

34. Samara, A., Andersen, P. T. \& Aro, A. R. Health Promotion and Obesity in the Arab Gulf States: Challenges and Good Practices. J Obes 2019, 4756260, https://doi.org/10.1155/2019/4756260 (2019).

35. Munger, K. L. et al. Childhood body mass index and multiple sclerosis risk: a long-term cohort study. Mult Scler 19, 1323-1329, https://doi.org/10.1177/1352458513483889 (2013).

36. Kavak, K. S. et al. Higher weight in adolescence and young adulthood is associated with an earlier age at multiple sclerosis onset. Mult Scler 21, 858-865, https://doi.org/10.1177/1352458514555787 (2015).

37. Mokry, L. E. et al. Obesity and Multiple Sclerosis: A Mendelian Randomization Study. PLoS Med 13, e1002053, https://doi. org/10.1371/journal.pmed.1002053 (2016). 
38. Novo, A. M. \& Batista, S. Multiple Sclerosis: Implications of Obesity in Neuroinflammation. Adv Neurobiol 19, 191-210, https://doi. org/10.1007/978-3-319-63260-5_8 (2017).

39. Guerrero-Garcia, J. J. et al. Multiple Sclerosis and Obesity: Possible Roles of Adipokines. Mediators Inflamm 2016, 4036232, https:// doi.org/10.1155/2016/4036232 (2016)

40. Jakimovski, D., Guan, Y., Ramanathan, M., Weinstock-Guttman, B. \& Zivadinov, R. Lifestyle-based modifiable risk factors in multiple sclerosis: review of experimental and clinical findings. Neurodegener Dis Manag 9, 149-172, https://doi.org/10.2217/nmt2018-0046 (2019).

41. Abdella, H. M., El Farssi, H. O., Broom, D. R., Hadden, D. A. \& Dalton, C. F. Eating Behaviours and Food Cravings; Influence of Age, Sex, BMI and FTO Genotype. Nutrients 11, https://doi.org/10.3390/nu11020377 (2019).

42. Sonestedt, E. et al. Fat and carbohydrate intake modify the association between genetic variation in the FTO genotype and obesity. Am J Clin Nutr 90, 1418-1425, https://doi.org/10.3945/ajcn.2009.27958 (2009).

43. Guilherme, J. et al. The A-allele of the FTO Gene rs9939609 Polymorphism Is Associated With Decreased Proportion of Slow Oxidative Muscle Fibers and Over-represented in Heavier Athletes. J Strength Cond Res 33, 691-700, https://doi.org/10.1519/ JSC.0000000000003032 (2019).

44. Heffernan, S. M. et al. Fat mass and obesity associated (FTO) gene influences skeletal muscle phenotypes in non-resistance trained males and elite rugby playing position. BMC Genet 18, 4, https://doi.org/10.1186/s12863-017-0470-1 (2017).

45. Edwards, T. \& Pilutti, L. A. The effect of exercise training in adults with multiple sclerosis with severe mobility disability: A systematic review and future research directions. Mult Scler Relat Disord 16, 31-39, https://doi.org/10.1016/j.msard.2017.06.003 (2017).

46. Rooney, S. et al. Physical activity is associated with neuromuscular and physical function in patients with multiple sclerosis independent of disease severity. Disabil Rehabil, 1-8, https://doi.org/10.1080/09638288.2019.1634768 (2019).

47. Brown, M. G. et al. Estimating typical multiple sclerosis disability progression speed from clinical observations. PLoS One 9, e105123, https://doi.org/10.1371/journal.pone.0105123 (2014).

48. Cerqueira, J. J. et al. Time matters in multiple sclerosis: can early treatment and long-term follow-up ensure everyone benefits from the latest advances in multiple sclerosis? Journal of neurology, neurosurgery, and psychiatry 89, 844-850, https://doi.org/10.1136/ jnnp-2017-317509 (2018).

49. Smemo, S. et al. Obesity-associated variants within FTO form long-range functional connections with IRX3. Nature 507, 371-375, https://doi.org/10.1038/nature13138 (2014).

50. Kurtzke, J. F. Disability rating scales in multiple sclerosis. Annals of the New York Academy of Sciences 436, 347-360 (1984).

51. Kurtzke, J. F. Historical and clinical perspectives of the expanded disability status scale. Neuroepidemiology 31, 1-9, https://doi. org $/ 10.1159 / 000136645(2008)$.

52. Gonzalez, J. R. et al. SNPassoc: an R package to perform whole genome association studies. Bioinformatics 23, 644-645, https://doi. org/10.1093/bioinformatics/btm025 (2007).

\section{Acknowledgements}

The Authors would like to thank the research sector at Kuwait University for their financial support under the grant MG03/19.

\section{Author contributions}

R.A.A. prepared the study design and performed the experiments and helped in writing the paper. R.A. Provided the samples and clinical data along with reading and approving the final manuscript. A.A. Prepared the study design and preformed the statistical analysis along with writing the manuscript.

\section{Competing interests}

The authors declare no competing interests.

\section{Additional information}

Correspondence and requests for materials should be addressed to A.A.-S.

Reprints and permissions information is available at www.nature.com/reprints.

Publisher's note Springer Nature remains neutral with regard to jurisdictional claims in published maps and institutional affiliations.

(c) (i) Open Access This article is licensed under a Creative Commons Attribution 4.0 International (c) License, which permits use, sharing, adaptation, distribution and reproduction in any medium or format, as long as you give appropriate credit to the original author(s) and the source, provide a link to the Creative Commons license, and indicate if changes were made. The images or other third party material in this article are included in the article's Creative Commons license, unless indicated otherwise in a credit line to the material. If material is not included in the article's Creative Commons license and your intended use is not permitted by statutory regulation or exceeds the permitted use, you will need to obtain permission directly from the copyright holder. To view a copy of this license, visit http://creativecommons.org/licenses/by/4.0/.

(C) The Author(s) 2019 